\title{
High Prevalence of Metallo-beta-lactamase Producing Acinetobacter baumannii Isolated From Two Hospitals of Tehran, Iran
}

\author{
Maryam Noori ${ }^{1}$, Abdollah Karimi ${ }^{2}$, Fatemeh Fallah ${ }^{2}$, Ali Hashemi ${ }^{1,}$, Shadi Alimehr ${ }^{2}$, Hossein \\ Goudarzi ${ }^{1}$, Shadi Aghamohammad ${ }^{1}$ \\ ${ }^{1}$ Department of Microbiology, Shahid Beheshti University of Medical Sciences, Tehran, IR Iran \\ 2 Pediatric Infections Research Center, Mofid Children's Hospital, Shahid Beheshti University of Medical Sciences, Tehran, IR Iran \\ ${ }^{*}$ Corresponding author: Ali Hashemi, Department of Microbiology,Shahid Beheshti University of Medical Sciences, Tehran,IRIran. Tel:+98-2123872556, E-mail: hashemi1388@yahoo.com.
}

Received: October 15, 2013; Revised: November 13, 2013; Accepted: December 21, 2013

\begin{abstract}
Background: Metallo-beta-lactamase (MBL)-producing Acinetobacter baumannii is a growing therapeutic concern, worldwide. Objectives: The aim of this study was to determine the frequency of SPM and IMP type genes among A. baumannii isolates from hospitalized patients in Loghman Hakim and Milad hospitals, Tehran-Iran from 2012 to 2013.

Patients and Methods: This study was conducted on $108 \mathrm{~A}$. baumannii isolates collected from two hospitals in Tehran, Iran. Antibiotic susceptibility tests were performed by Kirby-Bauer disc diffusion and Broth microdilution methods according to clinical laboratory standards institute (CLSI) guidelines, and combined disk diffusion test (CDDT) was performed for identification of metallo-beta-lactamases (MBLs). The blaIMP and blaSPM genes were detected by PCR and sequencing methods.

Results: The resistance of $A$. baumannii isolates against tested antibiotics were as follow: 108 (100\%) to cefotaxime, 103 (95.4\%) to ceftazidime, 99 (91.7\%) to meropenem, $99(91.7 \%)$ to imipenem, $44(40.7 \%)$ to gentamicin, $87(80.6 \%)$ to amikacin, $100(92.6 \%)$ to ciprofloxacin, 105 (95.7\%) to cefepime, 105 (97.2\%) to piperacillin, 103 (95.4\%) to piperacillin/tazobactam, 106 (98.1\%) to ampicillin/ sulbactam, $106(98.1 \%)$ to co-trimoxazole, $87(80.6 \%)$ to tetracycline and $1(1.8 \%)$ to colistin. Using combined disk diffusion test, it was found that out of 99 imipenem non-susceptible A. baumannii strains, 86 (86.86\%) were MBL producers. The prevalence of IMP-1 gene among metallo-beta-lactamase-producing A. baumannii isolates was 3 of 86 (3.48\%).

Conclusions: The prevalence of metallo-beta-lactamase-producing A. baumannii strains detected in this study is of great concern and highlights the need of infection control measures including antibacterial management and prompt identification of betalactamase-producing isolates.
\end{abstract}

Keywords: Acinetobacter baumannii; Drug Resistance; blaIMP-1

\section{Background}

Bacteria have the genetic ability to transfer and acquire antibiotic resistance factors. In recent years, the inappropriate use of antibiotics has led to drug resistance among bacteria, which is the cause of untreatable "superbugs" and high mortality rates throughout the world, particularly among patients with suppressed immunity. It is reported that about 25000 people in the E.U. and 63000 in the U.S. each year die from infectious diseases caused by multi-drug resistant (MDR) bacteria (1). A. baumannii is an important cause of nosocomial infections and has been associated with a wide variety of diseases in hospitalized patients in the intensive care units (ICU) and a leading cause of mortality and morbidity among hospitalized patients (2). Nowadays, increasing drug resistant rate among A. baumannii strains is a major concern worldwide (3). The most common mechanism of resistance is the production of $\beta$-lactamases, including enzymes of Ambler classes $\mathrm{A}, \mathrm{D}$ and $\mathrm{B}$, with their genes being often associated with mobile genetic elements such as plasmids (4). Carbapenem resistance caused by acquiring the metallo-betalactamases (MBLs) is considered to be more serious than other resistance mechanisms because MBLs can almost hydrolyse all beta-lactam antibiotics except monobactams (3). Furthermore, the MBL-encoding genes located on integrons can be disseminated easily from one bacterium to another. Many MBLs have been found in A. baumannii, including imipenemase (IMP), Sno Paolo metallo (SPM), Verona integron-encoded metallo-beta-lactamases (VIM), Seoul imipenemase (SIM), Japan, Kyorin University Hospital imipenemase (KHM), German imipenemase (GIM), New-Delhi metallo-beta-lactamase (NDM-1) and Australian imipenemase (AIM) $(5,6)$. The IMP-type enzymes, which were first detected in Japan in the late 1980s, have globally been reported in Enterobacteriaceae and in gram-negative non-fermenters (mostly in P. aeruginosa and Acinetobacter spp.) (3, 7). More than 20 different IMP allotypes belonging

Implication for health policy/practice/research/medical education:

The present study was conducted to determine the prevalence of SPM and IMP metallo- $\beta$-lactamases producing Acinetobacter baumannii isolated from intensive care units of two large Iranian public hospitals.

Copyright (c) 2014, Pediatric Infections Research Center. This is an open-access article distributed under the terms of the Creative Commons Attribution-NonCommercial 4.0 International License (http://creativecommons.org/licenses/by-nc/4.0/) which permits copy and redistribute the material just in noncommercial usages, provided the original work is properly cited. 
Noori M et al.

to various sublineages have been detected. The SPM-1 enzyme was the third type of acquired metallo-ß-lactamase discovered in the beginning of the 21st century in P. aeruginosa isolates from Brazil. So far, this enzyme is mostly confined to Brazil and P. aeruginosa. This low propensity to spread could be related to the different associated types of mobile genetic elements. SPM-1 has a very broad substrate specificity including carbapenems, cephalosporins, and penicillins (6).

\section{Objectives}

The aim of this study was to determine the frequency of SPM, and IMP type genes among A. baumannii isolates collected from hospitalized patients in the Loghman Hakim and Milad hospitals, Tehran-Iran from 2012 to 2013 years.

\section{Patients and Methods}

\subsection{Bacterial Identification}

From June 2012 to May 2013, one hundred and eight nonduplicate non-consecutive isolates of A. baumannii were recovered from blood, wound, urine, sputum, respiratory tract of patients from 2 hospitals (fifty-eight isolates belonging to Loghman Hakim and fifty isolates to Milad Hospital) in Tehran, Iran. The isolates were identified by conventional biochemical methods (2).

\subsection{Antimicrobial Susceptibility Testing}

Antimicrobial susceptibility test to imipenem (IPM: 10 $\mu \mathrm{g})$, meropenem (MEM: $10 \mu \mathrm{g})$, ceftazidime (CAZ: $30 \mu \mathrm{g}$ ), cefotaxime (CTX: $30 \mu \mathrm{g})$, amikacin (AK: $30 \mu \mathrm{g}$ ), piperacillin/tazobactam (PTZ: 100/10 $\mu \mathrm{g}$ ), piperacillin (PIP, $100 \mu \mathrm{g})$, ampicillin (AMP: $10 \mu \mathrm{g}$ ), tetracycline (TE: $10 \mu \mathrm{g}$ ), colistin sulphate (CT: $10 \mu \mathrm{g}$ ), ciprofloxacin (CIP: $5 \mu \mathrm{g}$ ), cefepime (FEP: $30 \mu \mathrm{g}$ ), trimethoprim-sulfamethoxazole (TS: $2.5 \mu \mathrm{g}$ ) and gentamicin (GEN: $10 \mu \mathrm{g}$ ) (Mast, UK), was performed by the Kirby-Bauer disk diffusion method on Mueller Hinton agar (Merck, Germany) based on clinical laboratory standards institute (CLSI) guidelines 2012 (8). Escherichia coli ATCC 25922 was used as quality control strain.

\subsection{Minimum Inhibitory Concentration (MIC)}

Strains resistant to imipenem, meropenem, ceftazidime, cefepime, cefotaxime and colistin using the disk diffusion test, were re-checked by the broth microdilution method according to the guidelines of the CLSI 2012 (8).

\subsection{Phenotypic Detection of $M B L$}

Combined disk diffusion test (CDDT) was performed for identification of MBLs by imipenem and meropenem (Mast Group, Merseyside, UK) alone and in combination with EDTA. We measured the inhibition zone at first, then compared to each other. An inhibition zone diameter dif- ference between the discs and discs + EDTA $\geq 7 \mathrm{~mm}$ were interpreted as a positive result for MBL production (7).

\subsection{DNA Extraction}

Total DNAs of the different bacterial isolates were extracted by the DNA extraction kit (Bioneer CompanyKorea, Cat. No. K-3032-2).

\subsection{Detection of blaIMP and blaSPM Genes by PCR}

PCR was used for screening of the blaIMP and blaSPM genes. The primers used for IMP and SPM were as follows: IMP-F (5'-GAAGGCGTTTATGTTCATAC-3') and TEM-R (5'-GTAAGTTTCAAGAGTGATGC-3') for blaIMP; and SPM-F (5' -CCTACAATCTAACGGCGACC- $\left.3^{-}\right)$, SPM-R (5 ${ }^{-}$-TCGCCGTGTCCAGGTATAAC- $3^{-}$) for blaSPM. The PCR mixture contained the DNA template, forward/reverse primers and master mix (Bioneer Company-Korea, Cat. No. K-2016). Amplification was carried out with the following thermal cycling conditions: 5 minutes at $94^{\circ} \mathrm{C}$ and 36 cycles of amplification consisting of 1 minute at $94^{\circ} \mathrm{C}, 1$ minute at $55^{\circ} \mathrm{C}$, and 1 minute at $72^{\circ} \mathrm{C}$, with 5 minutes at $72^{\circ} \mathrm{C}$ for the final extension. PCR product bands were analyzed after electrophoresis on a 1\% agarose gel at $95 \mathrm{~V}$ for 45 minutes in $1 \mathrm{X}$ TBE containing ethidium bromide and the result was checked under UV irradiation.

\subsection{Sequencing Method}

The PCR purification kit (Bioneer Co., Korea) was used to purify PCR products and sequencing was performed by the Bioneer company (Korea). The nucleotide sequences were analyzed with the Chromas 1.45 software and BLAST in NCBI.

\subsection{Statistical Analysis}

This research was a descriptive-application study. MINITAB 16 software was used for statistical analyses. The $P$ value and confidence of intervals were $<0.05$ and $95 \%$, respectively.

\section{Results}

Fifty-eight strains were isolated from Loghman Hakim Hospital (53.7\%) and fifty from Milad Hospital (46.29\%). Fifty-one strains were isolated from female patients (47.2 $\%$ ) and fifty-seven from males (52.8\%). Of the 108 isolates, 29 (26.9\%) were isolated from urine, 4 (3.7\%) from wound, 57 (52.8\%) from tracheal tube, 8 (7.4\%) from blood, 8 (7.4\%) from pleural fluid and 2 (1.9\%) from other samples. The age range of the patients was 1 to 90 years old. The isolates were obtained from patients in different age groups: 2-29 years $(n=10), 30-39(n=14), 40-49$ years $(n=21), 50-59$ years $(\mathrm{n}=16), 60-69(\mathrm{n}=24), 70-79$ years $(\mathrm{n}=17)$ and six isolates were isolated from patients more than eighty years old. The resistance of A. baumannii isolates to tested antibiotics was 108 (100\%) to cefotaxime, 103 (95.4\%) to ceftazidime, 99 
Noori M et al.

\begin{tabular}{llll}
\hline Table 1. Antimicrobial Susceptibility Test Results of 108 Isolated & Acinetobacter baumannii & \\
\hline Antibiotic & Resistant, No (\%) & Intermediate, No (\%) & Sensitive, No (\%) \\
\hline Gentamicin & $44(40.7)$ & $7(6.5)$ & $57(52.8)$ \\
\hline Ampicillin/sulbactam & $106(98.1)$ & $0(0.0)$ & $2(1.8)$ \\
\hline Amikacin & $87(80.6)$ & $4(3.7)$ & $17(15.7)$ \\
Imipenem & $99(91.7)$ & $3(2.8)$ & $6(5.6)$ \\
Cefotaxime & $108(100)$ & $0(0.0)$ & $0(0.0)$ \\
Cefepime & $105(95.7)$ & $2(1.8)$ & $1(1.8)$ \\
Piperacillin & $105(97.2)$ & $2(1.8)$ & $1(0.9)$ \\
Ciprofloxacin & $100(92.6)$ & $1(0.9)$ & $7(6.5)$ \\
Meropenem & $99(91.7)$ & $0(0.0)$ & $9(8.3)$ \\
Piperacillin/Tazobactam & $103(95.4)$ & $1(1.8)$ & $4(3.7)$ \\
Ceftazidime & $103(95.4)$ & $0(0.0)$ & $5(4.7)$ \\
Co-trimoxazole & $106(98.1)$ & $0(0.0)$ & $2(1.8)$ \\
Tetracycline & $87(80.6)$ & $9(8.3)$ & $12(11.1)$ \\
Colistin & $2(1.8)$ & $0(0.0)$ & $106(98.2)$ \\
\hline
\end{tabular}

\begin{tabular}{llll}
\hline Table 2. Minimum Inhibitory Concentration $(\mathrm{MIC})$ of Different Antimicrobial Agents Among 108 Acinetobacter baumannii Isolates \\
\hline & \multicolumn{2}{c}{ MIC $(\mu \mathbf{g} / \mathbf{m L})$} & MIC $_{90}$ \\
\cline { 2 - 4 } Antibiotics & Range & MIC $_{50}$ & 128 \\
\hline Meropenem & $1-256$ & 32 & 256 \\
Imipenem & $2-256$ & 128 & 512 \\
Ceftazidime & $2->512$ & 256 & 128 \\
Cefepime & $1-256$ & 64 & 512 \\
Cefotaxime & $2->512$ & 256 & 2 \\
\hline Colistin & $0.25-128$ & $\leq 1$ &
\end{tabular}

(91.7\%) to meropenem, 99 (91.7\%) to imipenem, 44 (40.7\%) to gentamicin, 87 (80.6\%) to amikacin, 100 (92.6\%) to ciprofloxacin, 105 (95.7\%) to cefepime, 105 (97.2\%) to piperacillin, 103 (95.4\%) to piperacillin/tazobactam, 106 (98.1\%) to ampicillin/sulbactam, 106 (98.1\%) to co-trimoxazole, $87(80.6 \%)$ to tetracycline and $1(1.8 \%)$ to colistin (Table 1$)$. The MIC of different antibiotics on A. baumannii isolates are shown in Table 2. By using the combined disk diffusion test (CDDT), it was found that among 99 imipenem non-susceptible A. baumannii strains, 86 (86.86\%) were MBL producers. The prevalence of IMP gene among metallo-beta-lactamaseproducing A. baumannii isolates was 3 of 86 (3.48\%), while bla (SPM) gene was not detected. Sequencing of PCR products showed conserved regions for the restriction sequence bla IMP-1 gene which was confirmed by BLAST in NCBI.

\section{Discussion}

A. baumannii is ubiquitous and has recently become one of the most important healthcare-associated infections in hospitals. Infection caused by this bacteria often leads to significant mortality and morbidity (9). The outbreaks of pan-drug resistant A. baumannii have rarely been detected. Although A. baumannii is an increasingly common nosocomial pathogen that can cause serious infections in intensive care units (ICU), most ICUs do not actively screen admissions for this pathogen (10). Resistance rate of isolates were as follows: 103 (95.4\%) to ceftazidime, 99 (91.7\%) to meropenem, 99 (91.7\%) to imipenem, 44 (40.7\%) to gentamicin, 87 (80.6\%) to amikacin, 100 (92.6\%) to ciprofloxacin, 105 (95.7\%) to cefepime, 105 (97.2\%) to piperacillin, 103 (95.4\%) to piperacillin/tazobactam, 106 (98.1\%) to ampicillin/sulbactam, 106 (98.1\%) to co-trimoxazole, 87 (80.6\%) to tetracycline and $1(1.8 \%)$ to colistin. Also, no sensitive isolate against cefotaxime was detected. So, the best coverage against the study isolates was obtained with colistin sulphate and gentamicin. Based on different studies, it is clear that emergence of resistant A. baumannii strains is increasing worldwide (2). These studies show that resistance rate of these strains is not only to beta lactams including the third generation of cephalosporins and carbapenemas but also to other drug divisions including aminoglycosides and fluoroquinolons. The other importance of these bacteria is related to their multi-drug resis- 
tance, which restricts their treatment. Shahcheraghi et al. reported that the resistance rates of $A$. baumannii strains to colistin and polymixin B were $12 \%$ and $3 \%$, respectively (2). Therefore it seems that these antibiotics are the only restricted antibiotics which are recommended for treatment of ICU patients in Iran during the recent days. Resistance to beta-lactams is related to various enzymes that are produced, including extended spectrum betalactamases (ESBLs) and metallo-beta-lactamases (MBLs), which belong to Ambler A and B division $(6,11)$. In this study by using the CDDT method, 86 (86.86\%) A. baumannii isolates were identified as MBL producers. Safari et al. reported that the resistance rates of $A$. baumannii isolates were $85 \%, 94 \%, 97 \%, 84 \%, 95 \%$ and $98 \%$ against imipenem, meropenem, ciprofloxacin, amikacin, piperacillin/tazobactam and cefotaxime, respectively. Results of E-test MBL illustrated that $99 \%$ of all isolates were MBL producers (12). Peymani et al. reported that among 63 carbapenem non-susceptible A. baumannii isolates, 31 (49\%) were found to be MBL producers. Of 31 MBL-producing isolates, 19 (61\%) carried the bla (IMP) gene and $9(29 \%)$ carried the bla (VIM) gene (13). Most of the time, the MBL producers can hydrolyze a wide range of antibiotics except aztreonam (14). Usually, restrictions in phenotypic methods makes researchers confirm phenotypic results by using molecular methods. On the other hand, there are different genes which encode the beta-lactamses. Among MBL genes, IMP is more important especially in Iran (15), however, its first report was from Japan in 1980 (7). The other gene is VIM, which was reported before from Ahwaz, another city of Iran (7). In our study, the IMP enzyme was identified only in three A. baumannii strains by using PCR and further sequencing which may be related to differences in the time of studies and consequently changes in antibiotics prescription or used primers. Also, other studies from Asia such as Malaysia and Lebanon detected IMP in A. baumannii isolates (6). In the case of SPM-1 gene which is responsible for MBL production, this gene was not detected by using PCR. However, Shahcheraghi et al. reported that 6 of 100 A. baumannii isolates were MBL producers and this gene was detected in all 6 strains (2). However, our results are not similar at one glance but deeper study showed the low frequency of this gene among Tehran isolated A. baumannii strains. Moreover, there is no report of frequency of this gene among Iranian $P$. aeruginosa isolates. However, in Brazil, 6 A. baumannii strains which were resistant to penicillins, cephalosporins and carbapenems contained the SPM-1 gene (2). Finally, by accurate MBL enzyme screening and further precise supervision of the hospital practitioners it will be possible to control the spread of multidrug resistant A. baumannii strains and decrease related infections specially in ICU patients.

The prevalence of ß-lactamase-producing isolates, and their isolation from life-threatening infections, is dramatically increasing worldwide. Intensity pressure for usage of antimicrobial drugs by patients resulted in eradication of normal flora and situation of MDR isolates substitution. This study showed that ß-lactamase producing A. baumannii strains are an emerging threat in ICUs and should be supervised by implementation of timely identification and strict isolation methods that will help to reduce their severe outcomes and mortality rate of patients.

\section{Acknowledgements}

We would like to thank the personnel of "Pediatric Infections Research Center” and Microbiology department of Shahid Beheshti University of Medical Sciences for their cooperation.

\section{Authors' Contribution}

Fatemeh Fallah and Ali Hashemi: analysis, interpretation of data and wrote the manuscript. Maryam Noori, Shadi Alimehr and Shadi Aghamohammad performed the microbiological and molecular studies. Abdollah Karimi and Hossein Goudarzi advised the search. All authors approved the final manuscript.

\section{Financial Disclosure}

The authors declare no competing financial interests.

\section{Funding/Support}

This work was financially supported by the Pediatric Infectious Research Center in Mofid Children's Hospital and Microbiology Department of Shahid Beheshti University of Medical Sciences, Tehran, Iran.

\section{References}

1. Dey D, Debnath S, Hazra S, Ghosh S, Ray R, Hazra B. Pomegranate pericarp extract enhances the antibacterial activity of ciprofloxacin against extended-spectrum beta-lactamase (ESBL) and metallo-beta-lactamase (MBL) producing Gram-negative bacilli. Food Chem Toxicol. 2012;50(12):4302-9.

2. Shahcheraghi F, Abbasalipour M, Feizabadi M, Ebrahimipour G, Akbari N. Isolation and genetic characterization of metallobeta-lactamase and carbapenamase producing strains of Acinetobacter baumannii from patients at Tehran hospitals. Iran J Microbiol. 2011;3(2):68-74.

3. Rahmati Roodsari M, Fallah F, Taherpour A, Hakemi Vala M, Hashemi A. Carbapenem-Resistant Bacteria and Laboratory Detection Methods. Arch Pediatr Infect Dis. 2013;1(4):188-91.

4. Fallah F, Taherpour A, Hakemi Vala M, Hashemi A. Global spread of New Delhi metallo-beta-lactamase-1(NDM-1). Iran J Clin Infect Dis. 2011;6(4):171-77.

5. Fallah F, Hakemi Vala M, Goudarzi H, Hashemi A, Taherpour A, Bigdeli Shamloo K, et al. Identification of extended-spectrumbetalactamases(ESBLs), metallo-beta-lactamases (MBLs), Amp-C and KPC ß-lactamases among Klebsiella pneumoniae isolated from adults and pediatric patients in Iran. African J Microbiol Res. 2013;7(25):3254-61.

6. Cornaglia G, Giamarellou H, Rossolini GM. Metallo-betalactamases: a last frontier for beta-lactams? Lancet Infect Dis. 2011;11(5):381-93.

7. Fallah F, Borhan RS, Hashemi A. Detection of bla(IMP) and 
Noori M et al.

bla(VIM) metallo-beta-lactamases genes among Pseudomonas aeruginosa strains. Int J Burns Trauma. 2013;3(2):122-4.

8. Clinical Laboratory Standards Institute. Performance standards for antimicrobial susceptibility testing: twenty second informational supplement.Wayne, PA: CLSI; 2012.

9. Fournier PE, Richet H.The epidemiology and control of Acinetobacter baumannii in health care facilities. Clin Infect Dis. 2006;42(5):692-9.

10. Lee BY, McGlone SM, Doi Y, Bailey RR, Harrison LH. Economic value of Acinetobacter baumannii screening in the intensive care unit. Clin Microbiol Infect. 2011;17(11):1691-7.

11. Owlia P, Azimi L, Gholami A, Asghari B, Lari AR. ESBL-and MBL-mediated resistance in Acinetobacter baumannii: a global threat to burn patients. Infez Med. 2012;20(3):182-7.

12. Safari M, Saidijam M, Bahador A, Jafari R, Alikhani MY. High
Prevalence of Multidrug Resistance and Metallo-beta-lactamase (MbetaL) producing Acinetobacter Baumannii Isolated from Patients in ICUWards, Hamadan, Iran.J Res Health Sci.2013;13(2):162-7.

13. Peymani A, Nahaei MR, Farajnia S, Hasani A, Mirsalehian A, Sohrabi N, et al. High prevalence of metallo-beta-lactamase-producing acinetobacter baumannii in a teaching hospital in Tabriz, Iran. Jpn J Infect Dis. 2011;64(1):69-71.

14. Liu Z, Li W, Wang J, Pan J, Sun S, Yu Y, et al. Identification and characterization of the first Escherichia coli strain carrying NDM-1 gene in China. PLoS One. 2013;8(6): e66666.

15. Yousefi S, Farajnia S, Nahaei MR, Akhi MT, Ghotaslou R, Soroush $\mathrm{MH}$, et al. Detection of metallo-beta-lactamase-encoding genes among clinical isolates of Pseudomonas aeruginosa in northwest of Iran. Diagn Microbiol Infect Dis. 2010;68(3):322-5. 